\section{BMJ Open Respiratory Research}

\title{
Hospital-based multidisciplinary lung cancer care in Australia: a survey of the landscape in 2021
}

\author{
Fraser J H Brims (D , , ${ }^{1,2}$ Chellan Kumarasamy, ${ }^{1}$ Jessica Nash, ${ }^{3}$ Tracy L Leong, ${ }^{3,4}$ \\ Emily Stone, ${ }^{5,6}$ Henry M Marshall (i) ${ }^{7}$
}

\section{ABSTRACT}

Introduction Lung cancer is the leading cause of cancer death in Australia and has the highest cancer burden. Numerous reports describe variations in lung cancer care and outcomes across Australia. There are no data assessing compliance with treatment guidelines and little is known about lung cancer multidisciplinary team (MDT) infrastructure around Australia.

Methods Clinicians from institutions treating lung cancer were invited to complete an online survey regarding the local infrastructure for lung cancer care and contemporary issues affecting lung cancer.

Results Responses from 79 separate institutions were obtained representing $72 \%$ of all known institutions treating lung cancer in Australia. Most (93.6\%) held a regular MDT meeting although recommended core membership was only achieved for $42 / 73(57.5 \%)$ sites. There was no thoracic surgery representation in 17/73 (23.3\%) of MDTs and surgery was less represented in regional and low case volume centres. Specialist nurses were present in just $37 / 79$ (46.8\%) of all sites. Access to diagnostic and treatment facilities was limited for some institutions. IT infrastructure was variable and most sites $(69 \%)$ do not perform regular audits against guidelines. The COVID-19 pandemic has driven most sites to incorporate virtual MDT meetings, with variable impact around the country. Clinician support for a national data-driven approach to improving lung cancer care was unanimous.

Discussion This survey demonstrates variations in infrastructure support, provision and membership of lung cancer MDTs, in particular thoracic surgery and specialist lung cancer nurses. This heterogeneity may contribute to some of the well-documented variations in lung cancer outcomes in Australia.

\section{INTRODUCTION}

(C) Author(s) (or their employer(s)) 2022. Re-use permitted under CC BY-NC. No commercial re-use. See rights and permissions. Published by BMJ.

For numbered affiliations see end of article.

Correspondence to Professor Fraser J H Brims; fraser.brims@curtin.edu.au
Lung cancer is the fifth most commonly diagnosed cancer and the leading cause of cancer death in Australia. ${ }^{1}$ There were more than 13200 cases diagnosed in Australia in 2020. It has a $19 \%$ 5-year survival rate $^{1}$ as most patients present with incurable disease where $42 \%$ of cases are diagnosed at stage 4 (most advanced) and $29 \%$ of cases are not staged at all. ${ }^{2}$ Lung cancer accounts for $9.1 \%$ of all

\section{Key messages}

The adequacy, resources and infrastructure for treating lung cancer in Australia is not known and may be associated with variations in patient outcomes.

- This national survey highlights widespread deficiencies in staffing at multidisciplinary team (MDT) meetings and access to critical diagnostics and treatment modalities; just $47 \%$ of all institutions have a specialist nurse and $23 \%$ have no access to thoracic surgery at MDT.

- This service heterogeneity may contribute to some of the well-documented variations in lung cancer outcome in Australia, highlighting urgent need to address these shortfalls.

cancers but is responsible for $18 \%$ of deaths from all cancers in Australia and $18.6 \%$ of the cancer burden. ${ }^{1}$

Lung cancer diagnosis and care is becoming increasingly complex, with a greater array of diagnostics and therapies than ever before. There are clear, evidence-based national guidelines which outline the expected standards of care and infrastructure available for patients with lung cancer, through the Optimal Care Pathway for People with Lung Cancer (OCP) and the Lung Cancer Framework Principle of Best Practice. ${ }^{3} 4$ These guidelines recommend timely access to critical interventions such as positron emission tomography-CT (PET-CT), endobronchial ultrasound (EBUS), thoracic surgeons with expertise in lung cancer and consideration for clinical trials. Due to the complexities of lung cancer management, the guidelines also recommend that every patient be discussed by a lung cancer multidisciplinary team (MDT), regardless of the patient's geographic location. The OCP specifies MDT membership including a 'core' team, who attend all/most meetings either in person or remotely, and an extended team. ${ }^{3}$ 
Diagnostic and treatment delays worsen outcomes for lung cancer ${ }^{5}$ and outcomes vary across the country. ${ }^{2}$ Australian and international data indicate wide variances in care between centres even in standard, established therapies. ${ }^{6}$ Data from two states in Australia (Victoria and New South Wales) consistently demonstrate similar findings: variations in care and outcomes between Indigenous and non-Indigenous Australians, rural and metropolitan patients, public and private centres and those of differing socioeconomic status. ${ }^{78}$ At least some of these variations result from differing infrastructure to investigate and treat lung cancer across Australia's hospitals.

Identifying gaps in care and ways to improve service delivery requires data to drive changes in practice. There are no Australian data assessing compliance with the guidelines, nor national data on the quality of care or outcomes for lung cancer at the patient level. At the most basic level, understanding the 'who' and 'where' of lung cancer treatment provision is important, yet little is currently known about the infrastructure of lung cancer care around Australia. This study, therefore, aimed to identify institutions treating patients with lung cancer in Australia, and survey infrastructure support and multidisciplinary care.

\section{METHODS}

MDT lead clinicians at institutions from all states and territories across Australia were invited to complete a voluntary online Qualtrics questionnaire survey. We identified institutions and MDT Leads via multiple sources including the Lung Foundation Australia (LFA) website, ${ }^{9}$ national professional bodies, lung cancer clinical and research interest groups and personal networks. The survey was open from 22 January 2021 to 7 July 2021.

Only one response per institution was required to avoid duplication. Variables included: institution characteristics (public/private; metropolitan/regional); estimated numbers of new lung cancers seen annually; MDT frequency, MDT team composition (see table 1), meeting set-up (face-to-face; virtual); diagnostic and treatment services on-site, information technology (IT) support for MDT meetings; staff and infrastructure support; impact of the COVID-19 pandemic; attitudes towards a potential Australian lung cancer clinical quality registry (CQR). Responses to statement questions were structured using 0-10 Likert scales. Case volume was defined as low ( $\leq 99$ new cases annually) or high $(\geq 100$ new cases annually). A copy of the survey is provided as an online supplemental file.

\section{Patient and public involvement}

Consumer representation influenced the concept and planning for this project as part of a wider effort towards identifying and alleviating variations in outcomes for lung cancer in Australia.
Table 1 Australian optimal care pathway for people with lung cancer recommended MDT membership ${ }^{3}$

\begin{tabular}{ll}
\hline Core members $^{*}$ & Extended members \\
\hline Care coordinator & Clinical psychologist \\
$>$ Medical oncologist & Clinical trials coordinator \\
Nuclear medicine & Dietitian \\
physician & General practitioner \\
Nurse (with appropriate & Occupational therapist \\
expertise) & Palliative care specialist \\
$>$ Pathologist & Pharmacist \\
Radiation oncologist & Physiotherapist \\
$>$ Radiologist/imaging & Psychiatrist \\
specialists & Social worker \\
$>$ Respiratory physician & \\
$>$ Thoracic surgeon & \\
\end{tabular}

${ }^{*}$ Core members of the multidisciplinary team are expected to attend most multidisciplinary team meetings either in person or remotely.

MDT, multidisciplinary team.

\section{Statistical analysis}

Adequate core data were defined as confirming the name of the institution that treats lung cancer and if the institution was regional or metropolitan, and public or private. Descriptive analysis was performed on the continuous variables of interest and frequencies were assessed for the dichotomised variables. The differences in categorical variables were analysed using the $\chi^{2}$ test. Qualitative data were analysed and grouped by themes, for instance, functioning and infrastructure issues for MDT and positive and negative impacts from the COVID-19 pandemic on the running of the MDT. Likert scales were analysed in accordance with standard approaches with the most frequent (mode) and/or median responses identified. Data were analysed using SPSS Statistics, V.24 (SPSS).

\section{RESULTS}

The LFA MDT database identified 86 institutions with a lung cancer MDT. The survey received a total of 96 responses, 13 were excluded due to incomplete survey data (in all cases respondents restarted and completed a separate response) and 4 were removed for duplication (multiple responders from same organisation). This left a total of 79 valid responses from separate institutions across all Australian states and territories. This included 23 institutions that were not included on the LFA database. The possible denominator for lung cancer MDTs in Australia could therefore reach 109 centres, suggesting a survey response rate of at least $72.4 \%$ for Australian lung cancer MDTs.

A summary of the data is presented in table 2. The denominator for responses varied as not all questions were answered by every respondent. Fifty-five institutions identified themselves as metropolitan and 24 regional with state breakdown as follows: Victoria 26, New South Wales 23, Queensland 13, Western Australia 10, South 
Table 2 Summary of responses from each Australian institution identified as managing lung cancer patients about the functioning of the local lung cancer multidisciplinary team

\begin{tabular}{|c|c|c|c|}
\hline & Total n (\%) & Metropolitan n (\%) & Regional n (\%) \\
\hline Total no institutions* & 79 & 55 & 24 \\
\hline Public & $65(82.3)$ & $42(76.4)$ & $1(4.2)$ \\
\hline Private & $14(17.7)$ & $13(23.6)$ & $23(95.8)$ \\
\hline Has regular MDT & 73/78 (93.6) & $52 / 54(96.3)$ & $21 / 24(87.5)$ \\
\hline No regular MDT & $5 / 78$ & 2 & 3 \\
\hline \multicolumn{4}{|c|}{ Estimate annual lung cancer cases for all centres } \\
\hline $0-49$ & $8 / 78(10.3)$ & $4 / 54(7.3)$ & $4 / 24(16.7)$ \\
\hline $50-99$ & $15 / 78(19.2)$ & $8 / 54(14.5)$ & $7 / 24(29.2)$ \\
\hline $100-199$ & $27 / 78(34.6)$ & $18 / 54(32.7)$ & 9/24 (37.5) \\
\hline$>200$ & 28/78 (35.9) & $24 / 54(43.6)$ & 4/24 (16.7) \\
\hline Specialist lung cancer nurse for all centres & $37 / 79(46.8)$ & $27 / 55(49.1)$ & $10 / 24(41.7)$ \\
\hline If yes FTE (median) & 0.8 & 0.8 & 0.8 \\
\hline \multicolumn{4}{|l|}{ Frequency of MDT } \\
\hline Weekly & $49 / 71(69.0)$ & $38 / 52(73.1)$ & $11 / 19(42.1)$ \\
\hline Fortnightly & $22 / 71(31.0)$ & $14 / 52(26.9)$ & $11 / 19(57.9)$ \\
\hline Core MDT members per OCP guidelines & $42 / 73(57.5)$ & $31 / 53(58.5)$ & $11 / 20(55.0)$ \\
\hline \multicolumn{4}{|l|}{ QA against National guidelines } \\
\hline None & $15 / 71(21.1)$ & $13 / 52(25)$ & 2/19 (10.5) \\
\hline Very infrequent & $11 / 71(15.5)$ & 9/52 (17.3) & $2 / 19(10.5)$ \\
\hline Yes, ad hoc & 23/71 (32.4) & $16 / 52(30.8)$ & 7/19 (36.8) \\
\hline Yes, regularly & 22/71 (31.0) & 14/52 (26.9) & $8 / 19(42.1)$ \\
\hline
\end{tabular}

All data presented as $\mathrm{n}=\mathrm{N}(\%)$ unless otherwise stated.

${ }^{*}$ The denominator for responses varied as not all questions were answered by every respondent.

FTE, (fraction of) full time equivalent; MDT, multidisciplinary team; OCP, Optimal Care Pathway; QA, quality assurance.

Australia 2, Tasmania 2, Northern Territory 2 and Australian Capital Territory 1. Every public tertiary hospital in Australia responded. More than two thirds of sites $(70.2 \%)$ estimated an annual new caseload of $\geq 100$.

Five $(6.3 \%)$ institutions reported no MDT meeting, one institution did not comment on MDT status. Of these, $4 / 5$ were public, $3 / 5$ regional, $1 / 5$ reported a new annual caseload of 100-199 and 4/5 reported a new annual caseload $<50$. Most MDTs met weekly, but around half of regional and low volume centres met fortnightly.

\section{Personnel and MDT membership}

Lung cancer nurse specialists (LCNS) were employed in $37 / 79(46.8 \%)$ sites, with a slightly lower proportion employed in regional areas. For those institutions with an LCNS, the median full-time equivalent (FTE) was 0.8 (range 0.2-1.5). The total FTE for LCNS reported for all institutions across Australia was 37 (with 27 FTE within metropolitan sites). Just over a quarter (27.3\%) of low case volume sites reported having an LCNS.

Data regarding MDT membership were analysed for the 73 MDTs (figure 1). Of 73, $42(57.5 \%)$ sites fulfilled core membership, no site fulfilled full recommended membership. One site had no respiratory representation, one site had geriatric medicine representation. Thoracic surgery was represented at 56/73 (76.7\%) sites and was significantly more common in metropolitan than regional centres, $\left(45 / 53\right.$ vs $11 / 20, \chi^{2}$ statistic 7.27 , $\mathrm{p}=0.007)$. Low case volume sites $(\leq 99 /$ year $)$ had less representation from thoracic surgery, nuclear medicine, specialist nurses and administrative support (table 3 and figure $1 \mathrm{~B})$.

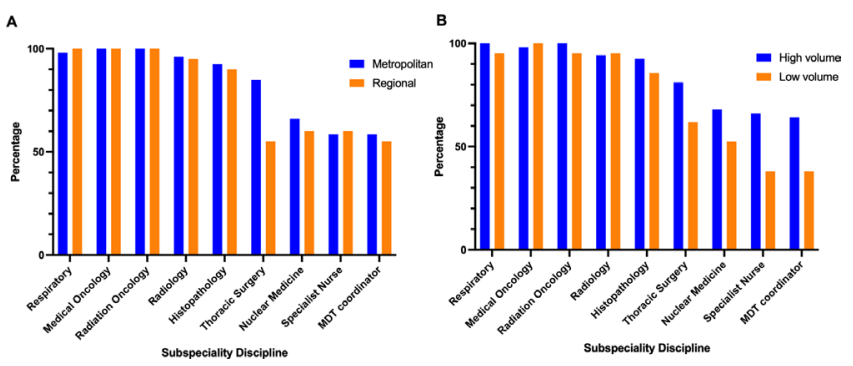

Figure 1 Per cent of lung cancer MDT attendance representation from subspeciality discipline in Australia, stratified by $(A)$ metropolitan and regional institutions, and $(\mathrm{B})$ high and low volume annual case numbers. MDT, multidisciplinary team. 
Table 3 Summary of responses from each Australian institution identified as managing lung cancer patients, stratified by high $(\geq 100)$ or low $(\leq 99)$ annual case volume

\begin{tabular}{|c|c|c|}
\hline & \multicolumn{2}{|c|}{ Annual cases $\mathrm{n} / \mathrm{N}(\%)$} \\
\hline & Low $(\leq 99)$ & $\operatorname{High}(\geq 100)$ \\
\hline Total no institutions $†$ & 23 & 55 \\
\hline Public & $15 / 23(65.2)$ & 49/55 (89.1) \\
\hline Private & $8 / 23(34.8)^{\star}$ & 6/55 (10.9) \\
\hline Metropolitan & $12 / 23(52.2)$ & $42 / 55(76.4)$ \\
\hline Regional & $11 / 23(47.8)$ & $13 / 55(23.6)$ \\
\hline Has regular MDT & 19/23 (82.6) & $54 / 55$ (98.2) \\
\hline No regular MDT & 4/23 (17.4) & $1 / 55(1.8)$ \\
\hline \multicolumn{3}{|l|}{ Frequency of MDT } \\
\hline Weekly & $7 / 18(38.9)$ & $42 / 53(76.4)$ \\
\hline Fortnightly & $11 / 18(47.8)^{\star}$ & $11 / 53(20.0)$ \\
\hline EBUS on site & 8/23 (34.8) & $50 / 55(90.9)$ \\
\hline Medical oncology on site & $20 / 23(87.0)$ & $53 / 55(96.4)$ \\
\hline Radiation oncology on site & $14 / 23(60.9)$ & $42 / 55(76.4)$ \\
\hline $\begin{array}{l}\text { Specialist lung cancer nurse } \\
\text { on site }\end{array}$ & $6 / 22(27.3)^{\star}$ & $33 / 53(62.3)$ \\
\hline If yes FTE (median) & 0.5 & 0.8 \\
\hline $\begin{array}{l}\text { Core MDT members per } \\
\text { OCP guidelines }\end{array}$ & 8/21 (38.1) & $34 / 53(64.2)$ \\
\hline $\begin{array}{l}\text { Full recommended MDT } \\
\text { attendance per OCP } \\
\text { guidelines }\end{array}$ & $0 / 21$ & $0 / 53$ \\
\hline \multicolumn{3}{|l|}{ QA against National guidelines } \\
\hline None & $7 / 17(41.2)$ & $9 / 53(16.4)$ \\
\hline Very infrequent & 3/17 (17.6) & $12 / 53(22.6)$ \\
\hline Yes, ad hoc & 4/17 (23.5) & 9/53 (17.0) \\
\hline Yes, regularly & 3/17 (17.6) & 23/53 (43.3) \\
\hline
\end{tabular}

All data presented as $\mathrm{n}(\%)$.

${ }^{*} \mathrm{P}<0.05$, compared with high volume.

†The denominator for responses varied as not all questions were answered by every respondent.

EBUS, endobronchial ultrasound; FTE, (fraction of) full time equivalent; MDT, multidisciplinary team; OCP, Optimal Care

Pathway; QA, quality assurance.

\section{Clinical infrastructure}

All 73 MDTs provided information on availability of diagnostic and treatment services. Medical oncology and palliative care services were available on all sites, with respiratory not available at one institution. PET-CT was available on site for 56/73 (76.7\%) and EBUS bronchoscopy for $58 / 73(79.5 \%)$. EBUS was less available in regional areas (metropolitan vs regional $46 / 53$ vs $12 / 20$, $\chi^{2}$ statistic $\left.6.38, \mathrm{p}=0.012\right)$. Thoracic surgery was on site for $56 / 73(76.7 \%)$ institutions, external beam radiotherapy $56 / 73(76.7 \%)$ and stereotactic ablative radiotherapy $48 / 73(65.8 \%)$. One regional site commented on an 'ad hoc service with minimal resources' and a private site reported 'fragmented care with no nurse'.

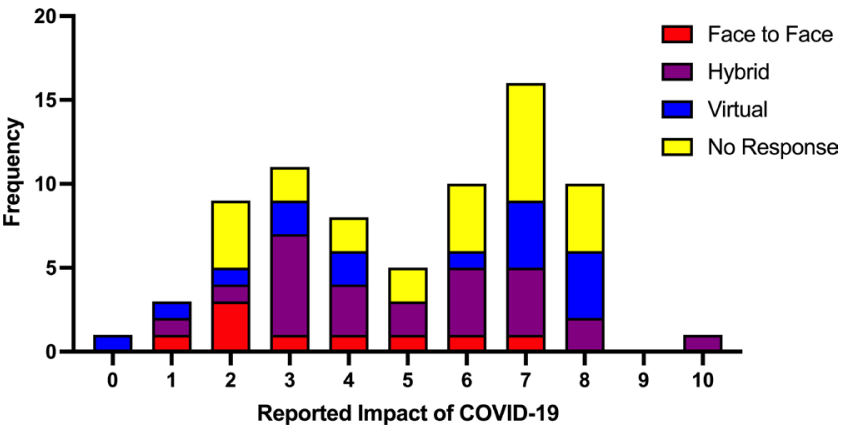

Figure 2 The reported impact of the COVID-19 pandemic on lung cancer services, stratified by changes to MDT meetings (face to face, hybrid or virtual). The scale represents 0 (no impact) to 10 (highest impact) from 75 institutions. MDT, multidisciplinary team.

\section{IT support}

Three MDTs reported no routine data collection and $6 / 73(8.2 \%)$ reported no routine IT support. For those MDTs which routinely collected data, $37 / 73(46.8 \%)$ used commercial third-party software and $27 / 73(34.1 \%)$ used standalone databases (eg, Microsoft Excel or Access). Over one-third of MDTs $(30 / 73,41.1 \%)$ reported no dedicated administrative support and data entry was performed by clinicians (consultants, fellows and registrars) in $53 / 73$ MDTs $(72.6 \%)$, or by an LCNS in $12 / 73$ MDTs $(16.4 \%)$.

\section{Multi-institution MDTs}

Collaboration across different institutions was evident: 9/73 sites commented on combined MDTs with larger institutions, 12/73 sites relying on other institutions for PET-CT and EBUS services and eight sites referring patients off site for radiation oncology and/or thoracic surgery. Further, 37/73 institutions reported having an LCNS on site, although 43/73 MDTs had LCNS representation.

\section{Impact from the COVID-19 pandemic}

There was a bimodal distribution of reported impact from the COVID-19 pandemic on lung cancer care (figure 2). Of 71,27 (38.0\%) MDTs reported instituting fully virtual meetings with $32(45.1 \%)$ initiating hybrid meetings. For fully virtual MDTs, five commented on increased participation by MDT members and one respondent commented that they "can see pathology slides and radiology better'; two sites noted reduced educational opportunities and less 'informal discussion' among members. There was no clear pattern for sites reporting lower or higher impact by state, location or case volume. Minimal or no change as a result of the pandemic was reported by 15 institutions, with nine sites continuing with face-to-face meetings. The impact of the pandemic was most evident in case presentations and service delivery. Twelve institutions commented on delays in case presentation and two that they had noted a stage shift to more advanced 
disease at presentation; one site noted an increase in referrals. Fourteen sites noted impact on service delivery with delays in access to diagnostic investigations and treatment. One institution commented that 'patients did not attend surgery' and one Victorian institution reported, 'significant impact \{on services\} during the second wave'.

\section{Attitudes towards a national lung cancer CQR}

Regarding a national lung cancer $\mathrm{CQR}$, 75 responses were received; there was strong support for a national registry (median score of 10 out of 10). Respondents indicated they felt the need for a national effort to improve lung cancer care is urgent (median score 9 out of 10).

\section{DISCUSSION}

This is the first Australia-wide survey of lung cancer services and care, representing up to $72 \%$ of all institutions known to treat lung cancer in Australia across all states and territories. Most responding sites were public and $70 \%$ reported seeing more than 100 cases per year. The results indicate important variations in the infrastructure for investigating and managing lung cancer.

No institutions had full recommended MDT membership, ${ }^{3}$ only $57 \%$ fulfilled recommended core membership and five institutions did not host a lung cancer MDT. Commonly missing groups included LCNSs (53.2\%), MDT co-ordinators $(42.5 \%)$, nuclear medicine $(35.6 \%)$ and thoracic surgery $(23.3 \%)$. Lung cancer MDT discussion improves a range of outcomes including staging, clinical decision making, better utilisation and uptake of treatments and survival ${ }^{10-12}$ and the quality of such meetings may be a reflection of the functioning of the wider culture of cancer care within a centre. National and international guidelines recommend MDTs in lung cancer care $^{3413}$ and, therefore, there is a clear need to improve the membership and infrastructure of lung cancer MDTs in Australia. Mitigating these MDT gaps, we observed a degree of collaboration across sites and note the recent widespread implementation of virtual meeting technologies. Similar to how telehealth has enabled remote patient-practitioner interactions, these technologies provide a mechanism to share resources and knowledge, and increase collaboration and referral pathways between low volume and high volume centres, which ultimately should translate to better patient outcomes. In addition, as discussed below, virtual meeting technologies may foster support and mentoring of newly established MDTs. ${ }^{14}$

The survey identified key gaps in on-site services and staffing. Although fewer than half of all institutions reported having an LCNS, particularly low case-volume institutions, the total FTE for all LCNS was better than previous estimates. ${ }^{9}$ Access to an LCNS is associated with increased treatment rates, reduced unplanned hospital admissions and improved addressing of patient needs including complex case navigation and emotional and psychological support. ${ }^{15-17}$ Specialist cancer nurses in
Australia are well recognised in breast and prostate cancer with an estimated 400 breast cancer nurses and at least 50 prostate cancer nurses in Australia. ${ }^{18} 19$ Federal commitment to fund five FTE LCNS in the 2021 Budget is welcome, but considering the burden of disease from lung cancer in Australia, the need for a significant uplift in the number of specialist lung cancer nurses remains.

Nearly one-quarter of MDTs (23\%) had no thoracic surgery representation despite the direct influence of surgical resection rates on survival in lung cancer. ${ }^{2021}$ The initial National Lung Cancer Audit reports for England and Wales demonstrated considerable variation in access to thoracic surgery, with subsequent changes leading to notable improvements resection rates. ${ }^{22}$

Site location was associated with infrastructure variations. Collaboration across sites appears to address some gaps for MDTs without on-site access to key investigations and treatments, however, regional and low volume centres demonstrated likely clinically important differences in access to EBUS bronchoscopy and thoracic surgery. Low volume sites reported less representation at MDT from thoracic surgery, nuclear medicine, specialist nurses and administrative support, and less frequent MDT meetings. This may worsen outcomes including survival, ${ }^{22} 23$ particularly for early stage lung cancer. ${ }^{24}$ Time delays may also affect regional patients due to travel required for investigations and/or curative treatment.

This survey identified 23 institutions not documented in the LFA database. Of these, $70 \%$ were public hospitals, $48 \%$ low volume centres $(0-49$ cases a year) and 10 regional centres. This likely, at least in part, represents expanding health services within local health networks across different jurisdictions. In turn, this reflects the dynamic landscape of lung cancer care, as new MDTs are established and grow to serve changing populations. Although intermittent snapshots of MDT care are of interest and paint a broad picture, they are unlikely to drive better patient outcomes in themselves. Rapid advances in lung cancer therapies, superimposed on continuously evolving demographics, mean that a 'realtime' national CQR should be the goal. Increased healthcare access for patients with lung cancer is undoubtedly important, and the establishment of new MDTs is welcome. At the same time, healthcare resource planning should be cognisant of research from other areas of medicine (including lung cancer surgery) demonstrating that high case volume is associated with better outcomes across a wide range of procedures and conditions. ${ }^{25} 26$ Although UK data demonstrates a correlation between quality of lung cancer services and 1-year survival rates, ${ }^{27}$ evidence to determine optimal lung cancer MDT case volumes is lacking, A recent consensus statement from Spain describes quality indicator requirements for a lung cancer MDT but does not make recommendations on case volume. ${ }^{28}$ This supports the urgent need to assess and ensure the quality of lung cancer services is adequate across all centres in Australia, to be able to track MDT 
delivery and outcomes over time and to support newly established MDTs.

The COVID-19 pandemic has driven innovations in models of care. Most centres had transitioned to either hybrid or fully virtual meetings, with only 12 sites continuing face-to-face meetings, some citing benefit and some drawback(s) with these approaches. This survey was not designed to investigate the impact in detail, although the free-text comments supported other recent reports that have demonstrated a decline in diagnostic procedures performed for lung cancer along with lower rates of diagnosis, reduction in clinical trial participation and increased 30-day mortality. ${ }^{14} 29$

It is perhaps surprising that many centres do not practice regular quality assurance (QA) against accepted standards of care. Fewer than one-third of MDTs reported a regular QA process. This survey identifies a number of areas which could be targeted to improve adherence to national and international standards of care for lung cancer. $^{3}{ }^{4}$ For example, dedicated MDT coordinators and commercial databases with IT support would likely improve data integrity. Clinicians clearly recognise the limitations in the data systems at present, and the value that a high-quality, uniform and linked data platform could provide. ${ }^{30}$ In this survey, the support for a national approach to auditing lung cancer care in Australia was strong, unanimous and urgent.

This survey has important limitations to consider. To reduce the volume of free text, the survey was designed with specific, focused questions, restricting the flexibility and depth of responses. To avoid duplication, the responses were not anonymised, which may have introduced responder bias. The survey did not specifically reference a guideline as a gold standard (but listed the full possible extended MDT membership). The second edition of the OCP for people with lung cancer was published June $2021^{31}$ and this survey closed on 7 July 2021, suggesting there will have been little confusion with the updated guidelines. Further, the suggested core MDT membership has not changed in the latest version.

The strength of this survey is its reach - likely representing over at least $70 \%$ of all institutions treating lung cancer in Australia. It represents an important first step towards improving lung cancer care. Multidisciplinary models of care overcome barriers to treatment, improve adherence to guidelines and are more likely to improve the quality of care for patients. ${ }^{11}$ The data from this survey provide a basis for review of Australian lung cancer services to improve collaboration across sites, reduce delays and improve access to timely investigation and treatment. Virtual platforms could improve MDT attendance and fill gaps in subspecialties across sites. The survey has quantified two major gaps in expertise-access to thoracic surgery support at MDTs and the lack of LCNS at almost half of all sites-both require urgent attention.

This survey of front-line clinicians who provide lung cancer care in Australia demonstrates variations in infrastructure support, provision and membership of lung cancer MDTs. This service heterogeneity may contribute to some of the well-documented variations in lung cancer outcome in Australia. Our survey respondents identified critical access issues to thoracic surgery and specialist lung cancer nurses and, for the first time, strong and unanimous support for a national CQR.

\section{Author affiliations}

${ }^{1}$ Curtin Medical School, Curtin University, Perth, Western Australia, Australia ${ }^{2}$ Department of Respiratory Medicine, Sir Charles Gairdner Hospital,

Nedlands, Western Australia, Australia

${ }^{3}$ Department of Respiratory and Sleep Medicine, Austin Health, Heidelberg, Victoria, Australia

${ }^{4}$ Personalised Oncology Division, Walter and Eliza Hall Institute of Medical Research, Parkville, Victoria, Australia

${ }^{5}$ Department of Respiratory Medicine, St Vincent's Hospital, Sydney, New South Wales, Australia

${ }^{6}$ St Vincent's Clinical School, University of New South Wales, Sydney, New South Wales, Australia

${ }^{7}$ Thoracic Research Centre, University of Queensland, Brisbane, Queensland, Australia

Contributors FB: concept, design, data collection, analysis, interpretation, writing and guarantor for overal content; CK: data analysis and writing; JN: data analysis and writing; TLL: concept, design, interpretation, writing; ES: concept, design, interpretation, writing; HMM: concept, design, interpretation, writing.

Funding The authors have not declared a specific grant for this research from any funding agency in the public, commercial or not-for-profit sectors.

Competing interests None declared.

Patient consent for publication Not applicable.

Ethics approval Curtin University Human Research Ethics approved the project (reference HRE2021-0031).

Provenance and peer review Not commissioned; externally peer reviewed.

Data availability statement All data relevant to the study are included in the article or uploaded as online supplemental information.

Supplemental material This content has been supplied by the author(s). It has not been vetted by BMJ Publishing Group Limited (BMJ) and may not have been peer-reviewed. Any opinions or recommendations discussed are solely those of the author(s) and are not endorsed by BMJ. BMJ disclaims all liability and responsibility arising from any reliance placed on the content. Where the content includes any translated material, BMJ does not warrant the accuracy and reliability of the translations (including but not limited to local regulations, clinical guidelines, terminology, drug names and drug dosages), and is not responsible for any error and/or omissions arising from translation and adaptation or otherwise.

Open access This is an open access article distributed in accordance with the Creative Commons Attribution Non Commercial (CC BY-NC 4.0) license, which permits others to distribute, remix, adapt, build upon this work non-commercially, and license their derivative works on different terms, provided the original work is properly cited, appropriate credit is given, any changes made indicated, and the use is non-commercial. See: http://creativecommons.org/licenses/by-nc/4.0/.

ORCID IDs

Fraser J H Brims http://orcid.org/0000-0002-6725-7535

Henry M Marshall http://orcid.org/0000-0002-9626-8014

\section{REFERENCES}

1 Cancer Australia. Lung cancer statistics Canberra, 2019. Available: https://lung-cancer.canceraustralia.gov.au/statistics [Accessed 04 Apr 2019].

2 National Cancer Control Indicators. Lung cancer strawberry hills. NSW: Cancer Australia, 2020. https://ncci.canceraustralia.gov.au/ cancer-types/lung-cancer

3 Cancer Council. Optimal care pathway for people with lung cancer, 2018. Available: https://www.cancer.org.au/content/ocp/health/ optimal-care-pathway-for-people-with-lung-cancer-june-2016

4 Lung Cancer Framework. Principles for best practice lung cancer care in Australia. Strawberry Hills, NSW: Cancer Australia, 2018. 
5 Rogers TK. Minimising diagnostic delay in lung cancer. Thorax 2019;74:319-20.

6 Wang T, Nelson RA, Bogardus A, et al. Five-year lung cancer survival: which advanced stage nonsmall cell lung cancer patients attain long-term survival? Cancer 2010;116:1518-25.

7 Roder D, You H, Baker D, et al. Using linked lung cancer registry and hospital data for guiding health service improvement. Asia Pacific Journal of Health Management 2016;11:65-75.

8 Wah W, Stirling RG, Ahern S, et al. Association between receipt of Guideline-Concordant lung cancer treatment and Individual- and Area-Level factors: a spatio-temporal analysis. Cancer Epidemiol Biomarkers Prev 2020;29:2669-79.

9 Lung Cancer Multidisciplinary Team Milton, QLD. Lung Foundation Australia, 2020. Available: https://lungfoundation.com.au/lungcancer-mdt/ [Accessed 22 Jan 2021].

10 Denton E, Conron M. Improving outcomes in lung cancer: the value of the multidisciplinary health care team. J Multidiscip Healthc 2016;9:137.

11 Stone E, Rankin N, Kerr S, et al. Does presentation at multidisciplinary team meetings improve lung cancer survival? Findings from a consecutive cohort study. Lung Cancer 2018;124:199-204.

12 Lin T, Pham J, Paul E, et al. Impacts of lung cancer multidisciplinary meeting presentation: drivers and outcomes from a population registry retrospective cohort study. Lung Cancer 2021;163:69-76.

13 Gaga M, Powell CA, Schraufnagel DE, et al. An official American thoracic Society/European respiratory Society statement: the role of the pulmonologist in the diagnosis and management of lung cancer. Am J Respir Crit Care Med 2013;188:503-7.

14 Leong TL. Delayed access to lung cancer screening and treatment during the COVID-19 pandemic: are we headed for a lung cancer pandemic? Respirology 2021;26:145-6.

15 McLaughlin-Barrett S. Outnumbered yet needed: the lung cancer nurse specialist. Respirology 2020;25:1027-8.

16 Stewart I, Khakwani A, Hubbard RB, et al. Are working practices of lung cancer nurse specialists associated with variation in peoples' receipt of anticancer therapy? Lung Cancer 2018;123:160-5.

17 Tod AM, Redman J, McDonnell A, et al. Lung cancer treatment rates and the role of the lung cancer nurse specialist: a qualitative study. BMJ Open 2015;5:e008587.

18 Sykes J, Ferri L, Kieman D. Prostate cancer specialist nurses in Australia - changing the face of supportive care through a national approach. The Australian Journal of Cancer Nursing 2014;15:22-8.
19 Ahern T, Gardner A, Courtney M. A survey of the breast care nurse role in the provision of information and supportive care to Australian women diagnosed with breast cancer. Nurs Open 2015;2:62-71.

20 Wah W, Stirling RG, Ahern S, et al. Influence of timeliness and receipt of first treatment on geographic variation in non-small cell lung cancer mortality. Int J Cancer 2021;148:1828-38.

21 Riaz SP, Lüchtenborg M, Jack RH, et al. Variation in surgical resection for lung cancer in relation to survival: population-based study in England 2004-2006. Eur J Cancer 2012;48:54-60.

22 Navani N, Nankivell M, Lawrence DR, et al. Lung cancer diagnosis and staging with endobronchial ultrasound-guided transbronchial needle aspiration compared with conventional approaches: an openlabel, pragmatic, randomised controlled trial. Lancet Respir Med 2015;3:282-9.

23 Yang C-FJ, Wang $\mathrm{H}$, Kumar A, et al. Impact of timing of lobectomy on survival for clinical stage la lung squamous cell carcinoma. Chest 2017; 152:1239-50.

24 Zuniga PVS, Ost DE. Impact of delays in lung cancer treatment on survival. Chest 2021;160:1934-58.

25 Halm EA, Lee C, Chassin MR. Is volume related to outcome in health care? A systematic review and methodologic critique of the literature. Ann Intern Med 2002:137:511-20.

26 Bernard A, Cottenet J, Mariet A-S, et al. Is an activity volume threshold really realistic for lung cancer resection? $J$ Thorac Dis 2018;10:5685-94.

27 Adizie JB, Khakwani A, Beckett P, et al. Impact of organisation and specialist service delivery on lung cancer outcomes. Thorax 2019;74:546-50.

28 Guirado M, Sanchez-Hernandez A, Pijuan L, et al. Quality indicators and excellence requirements for a multidisciplinary lung cancer tumor board by the Spanish lung cancer group. Clin Trans/ Oncol 2021. doi:10.1007/s12094-021-02712-8. [Epub ahead of print: 19 Oct 2021].

29 Reyes R, López-Castro R, Auclin E, et al. MA03.08 impact of COVID-19 pandemic in the diagnosis and prognosis of lung cancer. Journal of Thoracic Oncology 2021;16:S141-S41.

30 Brims F, Leong T, Stone E, et al. Variations in lung cancer care and outcomes: how best to identify and improve standards of care? Respirology 2021;26:1103-5.

31 Cancer Council Victoria. Optimal care pathway for people with lung cancer. 2nd ed. Melbourne, Victoria: Cancer Council Victoria, 2021. https://www.cancer.org.au/assets/pdf/lung-cancer-optimal-cancercare-pathway 